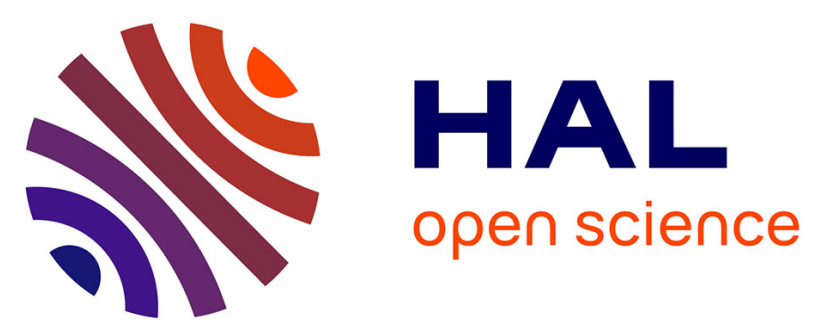

\title{
Temperature dependence of X-ray absorption and nuclear magnetic resonance spectra: probing quantum vibrations of light elements in oxides
}

Ruidy Nemausat, Christel Gervais, Christian Brouder, Nicolas Trcera, Amélie

Bordage, Cristina Coelho-Diogo, Pierre Florian, Aydar Rakhmatullin, Ion Errea, Lorenzo Paulatto, et al.

\section{To cite this version:}

Ruidy Nemausat, Christel Gervais, Christian Brouder, Nicolas Trcera, Amélie Bordage, et al.. Temperature dependence of X-ray absorption and nuclear magnetic resonance spectra: probing quantum vibrations of light elements in oxides. Physical Chemistry Chemical Physics, 2017, 19 (8), pp.6246 6256. 10.1039/C6CP08393E . hal-01480345

\section{HAL Id: hal-01480345 \\ https: / hal.sorbonne-universite.fr/hal-01480345}

Submitted on 1 Mar 2017

HAL is a multi-disciplinary open access archive for the deposit and dissemination of scientific research documents, whether they are published or not. The documents may come from teaching and research institutions in France or abroad, or from public or private research centers.
L'archive ouverte pluridisciplinaire HAL, est destinée au dépôt et à la diffusion de documents scientifiques de niveau recherche, publiés ou non, émanant des établissements d'enseignement et de recherche français ou étrangers, des laboratoires publics ou privés. 


\title{
Temperature dependence of $\mathbf{x}$-ray absorption and nu- clear magnetic resonance spectra: probing quantum vibrations of light element in oxides ${ }^{\dagger}$
}

\author{
Ruidy Nemausat, ${ }^{* a, b}$ Christel Gervais, ${ }^{b}$ Christian Brouder, ${ }^{a}$ Nicolas Trcera, ${ }^{c}$ Amélie \\ Bordage,$^{d}$ Cristina Coelho-Diogo, ${ }^{e}$ Pierre Florian, ${ }^{f}$ Aidar Rakhmatullin, ${ }^{f}$ Ion Errea,${ }^{g, h}$ \\ Lorenzo Paulatto, ${ }^{a}$ Michele Lazzeri ${ }^{a}$ and Delphine Cabaret $^{a}$
}

\begin{abstract}
A combined experimental-theoretical study on the temperature dependence of $x$-ray absorption near-edge structure (XANES) and nuclear magnetic resonance (NMR) spectra of periclase (MgO), spinel $\left(\mathrm{MgAl}_{2} \mathrm{O}_{4}\right)$, corundum $\left(\alpha-\mathrm{Al}_{2} \mathrm{O}_{3}\right)$, berlinite $\left(\alpha-\mathrm{AlPO}_{4}\right)$, stishovite and $\alpha$-quartz $\left(\mathrm{SiO}_{2}\right)$ is reported. Predictive calculations are presented when experimental data are not available. For these light-element oxides, both experimental techniques detect systematic effects related to quantum thermal vibrations which are well reproduced by density-functional theory simulations. In calculations, thermal fluctuation of the nuclei are included by considering nonequilibrium configurations according to finite-temperature quantum statistics at the quasiharmonic level. Nuclear quantum fluctuations on XANES and NMR spectroscopies are particularly sensitive to the coordination number of the probed cation. Furthermore, the relative importance of nuclear dynamics and thermal expansion is quantified over a large range of temperatures.
\end{abstract}

\section{Introduction}

X-ray absorption near-edge structure (XANES) and solid-state nuclear-magnetic resonance (NMR) spectroscopies are versatile probes of condensed matter. ${ }^{1,2}$ The combination of these two powerful techniques allows to thoroughly explore the electronic properties and local structure of materials. ${ }^{3-5}$ Moreover, recent breakthroughs in methodology and instrumentation provide a qualitative enhancement of experimental data. ${ }^{6-11}$ However, the

\footnotetext{
a Sorbonne Universités, UPMC Univ Paris 06, IMPMC, UMR CNRS 7590, 4 place Jussieu, F-75005 Paris, France.E-mail: ruidy.nemausat@impmc.upmc.fr

${ }^{b}$ Sorbonne Universités, UPMC Univ Paris 06, LCMCP, Collège de France, UMR CNRS 7574, 11 place Berthelot, F-75005 Paris, France

c Synchrotron SOLEIL, L'Orme des Merisiers, St Aubin, BP 48, F-91192 Gif sur Yvette, France

d ICMMO, Univ Paris Sud, Univ Paris-Saclay, UMR CNRS 8182, 15 rue du doyen Georges Poitou, F-91405 Orsay, France

e Sorbonne Universités, UPMC Univ Paris 06, IMPC, FR 2482, 4 place Jussieu, F-75005 Paris, France

${ }^{f}$ CEMHTI, UPR CNRS 3079, Univ Orléans, 1 Avenue de la Recherche Scientifique, F45071 Orléans, France

${ }^{g}$ Fisika Aplikatua 1 Saila, Bilboko Ingeniaritza Eskola, University of the Basque Country (UPV/EHU), Rafael Moreno "Pitxitxi" Pasealekua 3, 48013 Bilbao, Basque Country, Spain

${ }^{h}$ Donostia International Physics Center (DIPC), Manuel Lardizabal Pasealekua 4, 20018 Donostia/San Sebastián, Basque Country, Spain

$\dagger$ Electronic Supplementary Information (ESI) available: Calculation details, pseudopotential description and NMR anisotropic parameters. See DOI: $10.1039 / \mathrm{b} 000000 \mathrm{x} /$
}

use of accurate and appropriate theoretical tools is mandatory to fully interpret experiments. ${ }^{12-16}$

Most state-of-the-art simulation methods neglect nuclear degrees of freedom by assuming that atoms are fixed at their equilibrium positions. Nonetheless, even at zero temperature the influence of quantum fluctuations on nuclear motion remains as the zero-point motion, especially in the presence of light elements. At room temperature, the nuclear dynamics generates specific discrepancies between standard calculations and measurements, such as an additional pre-edge peak in the $K$-edge region of XANES spectra in light-element oxides ${ }^{17}$ and inaccurate assignment of NMR resonance peaks. ${ }^{18}$ In organic compounds, several studies already investigated the influence of atomic vibrations on XANES and NMR measurements. ${ }^{19-23}$ Besides, the impact of temperature is also noticeable in inorganic materials, in XANES preedge structure, ${ }^{24-26}$ chemical shifts, ${ }^{18,27}$ or electric-field gradient (EFG). ${ }^{28,29}$

Some attempts to theoretically model thermal effects in both spectroscopies emerged in the literature. Empirical methods based on either small displacements of the probed atom, ${ }^{30}$ or on averages of magnetic shielding tensors along specific orientations were proposed. ${ }^{31,32}$ Nuclear motion may also be considered via averages along molecular dynamics trajectories. ${ }^{33-37}$ This approach assumes that nuclei follow classical Newtonian trajectory of motion. Thus, it cannot describe phenomena associated to 
the quantum character of thermal fluctuations, which are especially relevant for light elements. Quantum thermal fluctuations may be taken into account by path integral molecular dynamics but the computational cost is an important limitation. ${ }^{38,39}$ Finitetemperature effects occurring in molecular crystals were successfully included via fragment-based techniques. ${ }^{40,41}$

In a recent paper, ${ }^{26}$ we presented an ab-initio method, based on the density-functional theory (DFT), ${ }^{42}$ to investigate the importance of quantum thermal vibrations in XANES and NMR spectroscopies. Following the work of Rossano et al., ${ }^{43}$ in the quasiharmonic approximation (QHA), ${ }^{44}$ thermal vibrations are modeled by constructing nonequilibrium structural configurations obeying the quantum statistics at finite temperature. This method was validated on $\mathrm{MgO},{ }^{26}$ a highly symmetric crystal, providing results closer to experiments than previous approaches. ${ }^{17,25,43}$

The current paper aims at generalizing this approach to several light-element oxides with various local cristallographic environments of the cations: periclase $(\mathrm{MgO})$, spinel $\left(\mathrm{MgAl}_{2} \mathrm{O}_{4}\right)$, corundum $\left(\alpha-\mathrm{Al}_{2} \mathrm{O}_{3}\right)$, berlinite $\left(\alpha-\mathrm{AlPO}_{4}\right)$, stishovite and $\alpha$-quartz $\left(\mathrm{SiO}_{2}\right)$. In these compounds, the probed atom is either 6 - or 4fold coordinated. Temperature-dependent experimental data are presented for both techniques and are compared to calculations. In NMR, when experimental data are not available, predictive calculations are provided. Thus, the influence of quantum thermal vibrations on NMR and XANES spectroscopies is shown and the relative importance of nuclear dynamics and thermal expansion is quantified. Furthermore, the influence of vibrations in both techniques is found to be sensitive to the coordination number of the probed cations.

The paper is structured as follows. Section 2 recalls the theoretical model used thereafter and gives computational details for XANES and NMR calculations. In Sec. 3, experimental details are provided. In Sec. 4, the experimental and theoretical results are presented, firstly focusing on experimental-theoretical comparison, then discussing the sensitivity to the coordination number of the probed cation. Finally, the conclusions of this work are drawn in Sec. 5

\section{Theoretical Approach}

\subsection{Quantum statistical mechanics}

The influence of thermal fluctuations is described by generating nonequilibrium configurations accounting for quantum statistics at finite temperature. Given a crystal, let us consider a supercell of $N$ nuclei, with $R_{i}^{\alpha}$ the position of the $i$ th nucleus along the $\alpha$ Cartesian coordinate. For simplicity, the two indexes are contracted into a single one $I$ such as $R_{i}^{\alpha}=R_{I}$, with $I=\{1, \ldots, 3 N\}$. Thereafter, the collective position vectors of the nuclei $\left(\overline{\mathbf{R}}=R_{1}, \ldots, R_{3 N}\right)$ is used. Within the Born-Oppenheimer approximation the total energy $E(\overline{\mathbf{R}})$ of the cell is considered as a function of the nuclear positions. The lattice-dynamical properties of a crystal are determined by the dynamical matrix, which reads at the $\Gamma$ point of the Brillouin zone

$$
D_{I J}=\left.\frac{1}{\sqrt{M_{I} M_{J}}} \frac{\partial^{2} E(\overline{\mathbf{R}})}{\partial u_{I} \partial u_{J}}\right|_{\mathbf{u}=\mathbf{0}},
$$

where $M_{I}$ is the mass of the $I$ th atom and $u_{I}$ is the displacement from the equilibrium position $\mathrm{R}_{I,(\mathrm{eq})}\left(R_{I}=R_{I,(\mathrm{eq})}+u_{I}\right)$. The frequency $\Omega_{\mu}$ and polarization vector $\mathbf{e}_{I, \mu}$ of the phonon mode $\mu$ are obtained from

$$
\sum_{J=1}^{3 N} D_{I J} e_{J \mu}=\Omega_{\mu}^{2} e_{I \mu}
$$

where $\sum_{I}\left|e_{I, \mu}\right|^{2}=1$. At a given temperature $T$, the probability $P(\overline{\mathbf{R}})$ of finding the crystal in the $\overline{\mathbf{R}}$ configuration is

$$
P(\overline{\mathbf{R}})=A \exp \left[-\sum_{\mu}^{\prime} \frac{\left(\sum_{I} \sqrt{M_{I}} e_{I \mu} u_{I}\right)^{2}}{2 a_{\mu}}\right]
$$

where $A$ is a normalization constant, $\sum_{\mu}^{\prime}$ is the sum on the $3 N-3$ modes with $\Omega_{\mu} \neq 0$ and

$$
a_{\mu}=\sqrt{\frac{\hbar}{2 \Omega_{\mu}} \operatorname{coth}\left(\frac{\hbar \Omega_{\mu}}{2 k_{B} T}\right)}
$$

is the normal length of the $\mu$ th mode of vibration at the $T$ temperature $\left(k_{B}\right.$ is the Boltzmann constant). Nuclear configurations are generated by Monte-Carlo sampling of $P(\overline{\mathbf{R}})$.

In practice, a set of configurations is generated as follows: for each $\mu$ mode, a set of $N_{c}$ random Gaussian numbers (with unit variance and zero expectation) $\left\{x_{\mu}^{i}\right\}_{i=1, N_{c}}$ is created. The nuclear positions collective vectors $\overline{\mathbf{R}}^{i}$ are then given by:

$$
R_{I}^{i}=R_{I,(\mathrm{eq})}^{i}+\sum_{\mu}^{\prime} \frac{1}{\sqrt{M_{I}}} e_{I \mu} a_{\mu} x_{\mu}^{i} .
$$

As in several applications, ${ }^{23,43}$ the quantum statistical average at temperature $T$ of any observable $O$ is computed via the following expression:

$$
O(T)=\int d \overline{\mathbf{R}} P(\overline{\mathbf{R}}) O(\overline{\mathbf{R}}) \simeq \frac{1}{N_{c}} \sum_{i=1}^{N_{c}} O\left(\overline{\mathbf{R}}^{i}\right)
$$

In this paper, $O$ is either the x-ray absorption cross section $\sigma(\hbar \omega)$, the NMR chemical shift $\delta$ or the quadrupolar coupling constant $C_{Q}$. Henceforth, this model is referred to as the QHA model. For more details refer to Refs. 26,45.

\subsection{Solid-state nuclear magnetic resonance}

For each nucleus, the isotropic value of the magnetic shielding tensor $\hat{\sigma}$ is evaluated as $\sigma_{\text {iso }}=\operatorname{tr}(\hat{\sigma}) / 3$. To compare with NMR experiments, the isotropic chemical shift $\delta_{\text {iso }} \approx \sigma_{\text {ref }}-\sigma_{\text {iso }}$ is considered, where $\sigma_{\text {ref }}$ is the isotropic shielding of a standard reference. The chemical shift $\delta(T)$ at finite temperature is obtained as in eqn (6):

$$
\delta_{\text {iso }}(T)=\frac{1}{N_{c}} \sum_{i=1}^{N_{c}} \delta_{\text {iso }}\left(\overline{\mathbf{R}}^{i}\right) .
$$

In the case of quadrupolar nuclei (nuclear spin $>1 / 2$ ), the nuclear quadrupole electric moment $Q$ interacts with the electricfield gradient (EFG) resulting in the quadrupolar interaction. ${ }^{8}$ Ordering the principal values of the EFG tensors $\hat{V}$ such as $\left|V_{z z}\right|>$ $\left|V_{x x}\right|>\left|V_{y y}\right|$, the magnitude of the quadrupolar interaction is quantified by the quadrupolar coupling constant $C_{Q}=e Q\left|V_{z z}\right| / h$, 
with $h$ the Planck constant and $e$ the elementary charge.

\subsection{X-ray absorption near-edge structure}

For a given $\overline{\mathbf{R}}$ configuration, in a single-electron approach, at the $K$ edge, the XANES cross-section in the electric dipole approximation is usually given by ${ }^{46}$

$$
\sigma(\hbar \omega ; \overline{\mathbf{R}})=4 \pi^{2} \alpha_{0} \hbar \omega \sum_{f}\left|\left\langle\psi_{f}|\hat{\mathbf{e}} \cdot \mathbf{r}| \psi_{1 s}\right\rangle\right|^{2} \delta\left(\varepsilon_{f}-\varepsilon_{1 s}-\hbar \omega\right),
$$

where $\hbar \omega$ and $\hat{\mathbf{e}}$ are the energy and polarization vector of the incident $\mathrm{x}$-ray beam, respectively, $\mathbf{r}$ the electron position and $\alpha_{0}$ the fine structure constant. The final $\left|\psi_{f}\right\rangle$ and initial $\left|\psi_{1 s}\right\rangle$ monoelectronic states have $\varepsilon_{f}$ and $\varepsilon_{1 s}$ for respective energies. Eqn (8) is derived by assuming that the atoms are fixed in a generic $\overline{\mathbf{R}}$ nuclear configuration, hence $\sigma$ is parametrized by $\overline{\mathbf{R}}$.

The rigorous expression of the cross section accounting for the total system of nuclei and electrons reads

$$
\sigma_{\mathrm{tot}}(\hbar \omega)=4 \pi^{2} \alpha_{0} \hbar \omega \sum_{f, j}\left|\left\langle\Psi_{f}^{j}|\hat{\mathbf{e}} \cdot \overline{\mathbf{r}}| \Psi_{g}^{0}\right\rangle\right|^{2} \delta\left(E_{f}^{j}-E_{g}^{0}-\hbar \omega\right),
$$

where, $\overline{\mathbf{r}}=\left\{\mathbf{r}_{1}, \ldots, \mathbf{r}_{N_{e}}\right\}$ is the collective position vector of the electrons, $j$ indexes the vibrational states, the $\Psi_{g}^{0}$ ground-state and $\Psi_{f}^{j}$ final state of the electrons plus nuclei total system and $E_{f}^{j}$ is the total energy in the $f$ and $j$ electronic and vibrational states, respectively.

In Ref. 26, we demonstrated that $\sigma_{\text {tot }}(\hbar \omega)$ can be expressed in the Born-Oppenheimer approximation, in a single-electron framework, as an average at the cost of neglecting the nuclear kinetic energy, by

$$
\sigma_{\text {tot }}(\hbar \omega)=\int d \overline{\mathbf{R}} P(\overline{\mathbf{R}}) \sigma(\hbar \omega ; \overline{\mathbf{R}}) .
$$

In eqn (10), $\sigma_{\text {tot }}$ is the average of individual configuration cross sections $\sigma(\hbar \omega ; \overline{\mathbf{R}})$, given by eqn (8), using a probability distribution that takes into account the temperature and vibrations frequencies in a form given by eqn (6).

\subsection{Computational details}

Calculations are performed in the DFT framework on the six different oxides reported in Table 1 . In all cases, the equilibrium structure (crystal-cell parameters and atomic positions) is the experimental one extracted from x-ray diffraction (XRD) analysis at various temperatures (see Refs. in Table 1). For each temperature, phonons are calculated for the corresponding experimental

Table 1 List of the studied compounds and temperature-dependent diffraction data references. The coordination number of the probed atom is indicated between parentheses

\begin{tabular}{llll}
\hline Mineral (Formula) & Sample type & Probed atom & Refs. \\
\hline Periclase $(\mathrm{MgO})$ & single crystal & $\mathrm{Mg}(6)$ & 47 \\
Spinel $\left(\mathrm{MgAl}_{2} \mathrm{O}_{4}\right)$ & powder & $\mathrm{Mg}(4), \mathrm{Al}(6)$ & 48,49 \\
Corundum $\left(\alpha-\mathrm{Al}_{2} \mathrm{O}_{3}\right)$ & single crystal & $\mathrm{Al}(6)$ & 49,50 \\
Berlinite $\left(\alpha-\mathrm{AlPO}_{4}\right)$ & powder & $\mathrm{Al}(4)$ & 51 \\
Stishovite $\left(\mathrm{SiO}_{2}\right)$ & polycrystal & $\mathrm{Si}(6)$ & 52 \\
$\alpha$-Quartz $\left(\mathrm{SiO}_{2}\right)$ & single crystal & $\mathrm{Si}(4)$ & 53,54
\end{tabular}

structure, allowing the generation of a set of various random nuclear configurations according to quantum-statistical mechanics rules (see Sec. 2.1). XANES spectra and NMR parameters are then calculated and averaged on these configurations (see Sec. 2.2 and 2.3). To quantify the relative importance of lattice dynamics with respect to thermal expansion, calculations are also performed on the experimental XRD structures, referred as to the thermal expansion only (TEO) in the following.

DFT calculations are performed using the QUANTUM ESPRESSO plane-wave pseudopotential distribution, ${ }^{55}$ particularly the $\mathrm{PW}$, PH, ${ }^{56,57}$ XSPECTRA ${ }^{58,59}$ and GIPAW ${ }^{60,61}$ packages. Stochastic nuclear configurations are generated in a large-enough supercell configuration (see $\mathrm{ESI}^{\dagger}$ ) with the sSCHA code. ${ }^{62,63}$

Perdew-Burke-Ernzerhof implementation of the exchangecorrelation functional ${ }^{64}$ and GIPAW reconstruction of coreelectron distributions ${ }^{60,61}$ are used. NMR calculations are performed using norm-conserving pseudopotentials, ${ }^{65}$ while ultrasoft ones are chosen for phonon and XANES calculations. ${ }^{66}$ For XANES, the absorbing atom pseudopotential is generated from an electronic configuration, from which one $1 s$ electron is removed. Other computational details (plane-wave cut-off, electronic sampling, pseudopotential details) are gathered in ESI. ${ }^{\dagger}$

The NMR reference shieldings $\sigma_{\text {ref }}$ are chosen so that experimental and calculated values match at room temperature. To highlight the trends, we focus on the chemical shifts variations from the room-temperature: $\Delta \delta_{\text {iso }}=\delta_{\text {iso }}(T)-\delta_{\text {iso }}(300 K)$. The $C_{Q}$ of ${ }^{17} \mathrm{O},{ }^{25} \mathrm{Mg}$ and ${ }^{27} \mathrm{Al}$ nuclei are calculated with $Q$ values taken from Ref. 67, by averaging individual configuration values. ${ }^{68}$ In each configuration, the chemical shift is computed for every atom sites. The number of configurations required to converge the averaged chemical shift of about $0.1 \mathrm{ppm}$ is around 10 .

In XANES simulations, for each temperature, the spectra are calculated in configurations including one $1 s$ full core-hole in a random absorbing-atom site. The absorbing atom is isolated from its periodic images by constructing a large-enough supercell. For all compounds, the number of configurations necessary to converge the XANES calculation is between 30 and 40. A corelevel shift is applied before comparing and averaging theoretical spectra as, in the pseudopotential approximation, only valenceelectrons are considered, hence the energy scale loses its physical meaning. 69

\section{Experimental Methods}

\subsection{Samples}

Six mineral oxides samples are investigated, where the probed atom is either 4- or 6-fold coordinated (Table 1). The corundum sample was already used in Ref. 70. The stishovite polycrystal comes from a high-pressure synthesis. ${ }^{71}$ For NMR measurements, only powder samples are considered. For XANES, resorting to single-crystal samples for $\alpha-\mathrm{Al}_{2} \mathrm{O}_{3}$ and $\alpha$-quartz allows polarized XANES measurements. For each compound, the maximum temperature explored remains below their respective phase-transition/fusion temperature. 


\subsection{Temperature-dependent experiments}

Temperature-dependent NMR measurements are recorded at the CEMHTI (Orléans, France). The experimental acquisitions are carried out using a Bruker Avance spectrometer operating at $19.8 \mathrm{~T}(850 \mathrm{MHz})$ and a static NMR probe designed to handle high temperatures. ${ }^{72}$ The boron-nitride (BN) sample holder is heated by a $\mathrm{CO}_{2}$ laser. Given the sensitivity of ${ }^{27} \mathrm{Al}$ nucleus and the expected variations of the chemical shifts compared to the static NMR peak width, ${ }^{27} \mathrm{Al}$ in $\alpha-\mathrm{AlPO}_{4}$ is the best suitable candidate. A magic-angle spinning NMR probe is required to increase the resolution in $\alpha-\mathrm{Al}_{2} \mathrm{O}_{3}$ and $\mathrm{SiO}_{2}$ polymorphs. We also present the chemical shifts of ${ }^{25} \mathrm{Mg}$ and ${ }^{17} \mathrm{O}$ in $\mathrm{MgO}$ from Ref. 27.

X-ray absorption experiments are performed at the LUCIA beamline from SOLEIL french synchrotron facility (Saint-Aubin, France). ${ }^{73}$ For each compound, the incident energy ranges are selected to include the $K$ edge of either $\mathrm{Mg}, \mathrm{Al}$ or $\mathrm{Si}$. The experimental chamber pressure is set between $10^{-7}$ and $10^{-6}$ mbar. The temperature of the sample is set using a BN furnace and measured with a chromel-alumel thermocouple. The spectra are recorded in fluorescence with a four element silicon drift diode detector, protected from infrared and visible radiations by a beryllium window. To improve the signal to noise ratio, each point is obtained after an acquisition time of $5 \mathrm{~s}$ and several spectra are measured for each temperature. The normalization and self-absorption correction procedures are conducted as described in Ref. 25. For $\alpha$ - $\mathrm{Al}_{2} \mathrm{O}_{3}$ and $\alpha$-quartz samples, the two $\sigma_{\perp}$ and $\sigma_{/ /}$polarized spectra, corresponding respectively to ê parallel and perpendicular to the threefold symmetry axis of the crystals, were successively measured. Experimental $\mathrm{Mg}$ and $\mathrm{Al} K$-edges XANES spectra in $\mathrm{MgO}$ and $\alpha-\mathrm{Al}_{2} \mathrm{O}_{3}$, respectively, have already been published elsewhere. ${ }^{25,26}$

\section{Results and Discussion}

\subsection{Experimental and calculation comparison}

Fig. 1 compares experimental and calculated temperaturedependent chemical shifts of ${ }^{25} \mathrm{Mg}$ and ${ }^{17} \mathrm{O}$ in $\mathrm{MgO}$ and ${ }^{27} \mathrm{Al}$ in $\alpha-\mathrm{AlPO}_{4}$. From 300 to $1300 \mathrm{~K}$, an increase of about 2 and $12 \mathrm{ppm}$ is observed for experimental ${ }^{25} \mathrm{Mg}$ and ${ }^{17} \mathrm{O} \delta_{\text {iso }}$ values, respectively. On the other hand, experimental ${ }^{27} \mathrm{Al}$ chemical shift decreases with temperature, by about 4 ppm between 300 and $800 \mathrm{~K}$. In both cases, simulations are in good agreement with experiments over the investigated range of temperatures. As detailed in Refs. 26,74, the remaining discrepancies between calculated and experimental slopes come mainly from deficiency of GGA. It is known that GGA slightly overestimates the interatomic distances of solids and underrates phonon frequencies. ${ }^{75}$ In the present work, experimental lattice parameters are used to balance this effect. The use of GGA functionals more advanced than PBE might also enhance the agreement. ${ }^{76,77}$

Usually, a linear thermal behavior of NMR parameters is assumed. ${ }^{19,27}$ However, the temperature dependence of the chemical shift clearly shows two regimes. The linear correlation observed above a saturation temperature $T_{S}$ reflects a classical meanfield behavior of the Landau-type. ${ }^{78}$ Between $0 \mathrm{~K}$ and $T_{s}$ a quantum saturation appears, where the chemical shift becomes inde-
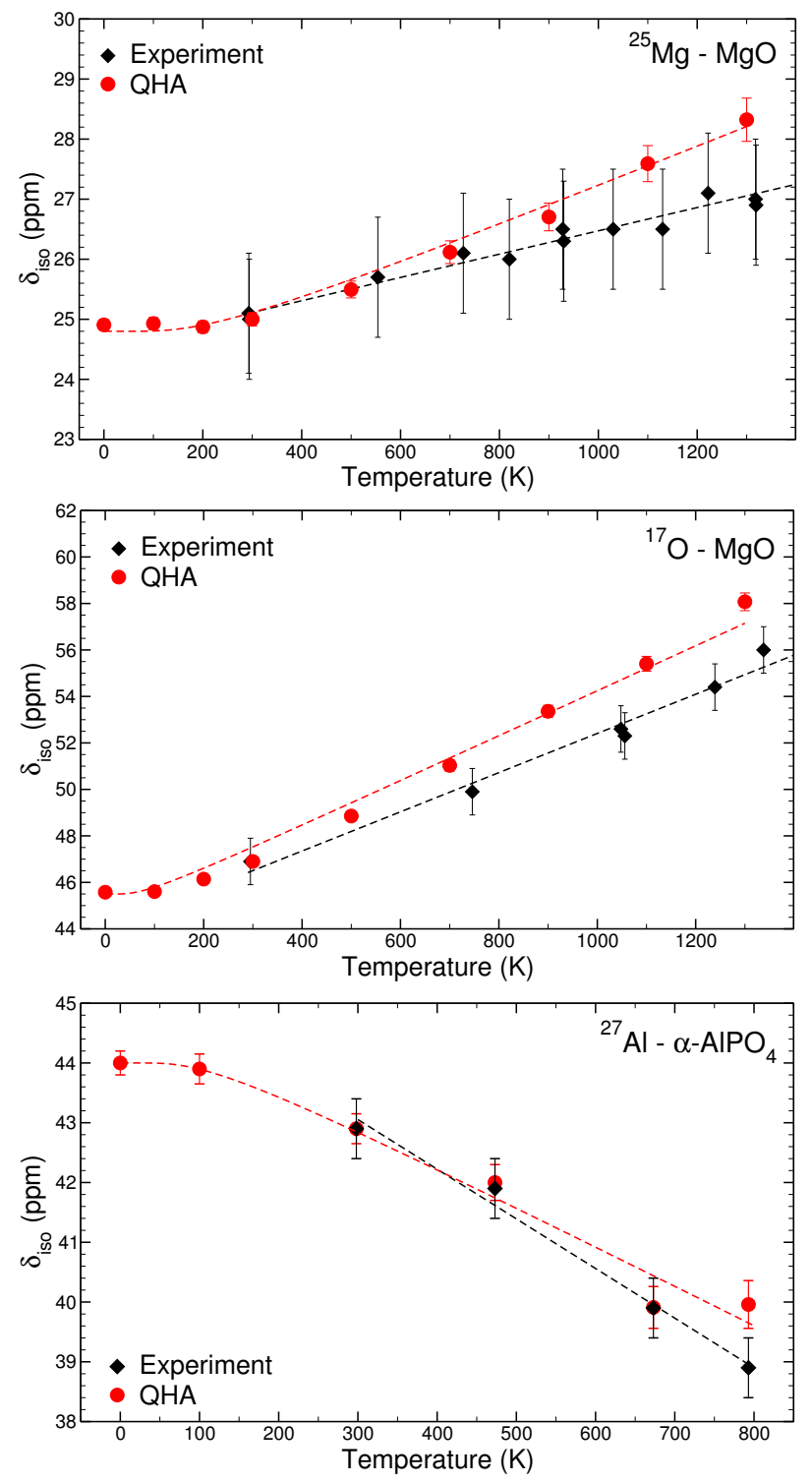

Fig. 1 Experimental and calculated temperature dependence of ${ }^{25} \mathrm{Mg}$ and ${ }^{17} \mathrm{O}$ chemical shifts in $\mathrm{MgO}$ and of ${ }^{27} \mathrm{Al}$ chemical shifts in berlinite $\left(\alpha\right.$-AlPO $\left.{ }_{4}\right) .{ }^{25} \mathrm{Mg}$ measurements come from Ref. 27 , whereas ${ }^{27} \mathrm{Al}$ ones are original datasets. The red dashed line is the best fit using eqn (11).

pendent of the temperature. Therefore, the chemical shift is fitted using

$$
\delta(T)=A+B \operatorname{coth}\left(\frac{2 T_{s}}{T}\right),
$$

with $A$ and $B$ the fitting parameters, as in Ref. 79. The resulting saturation temperatures are given in ESI. ${ }^{\dagger}$ Thereby, linear extrapolations of the chemical shifts to $0 \mathrm{~K}$ prove to be incorrect in inorganic materials, which confirms that temperature-dependent firstprinciples calculations are mandatory. Low temperature measurements of NMR parameters are expected to confirm this conclusion.

Fig. 2 compares the experimental and calculated temperaturedependent XANES spectra at the $K$ edge of the studied cations in oxides. The collection of XANES spectra generalizes the thermal behavior that we have already discussed at the $K$ edge of $\mathrm{Mg}$ and 

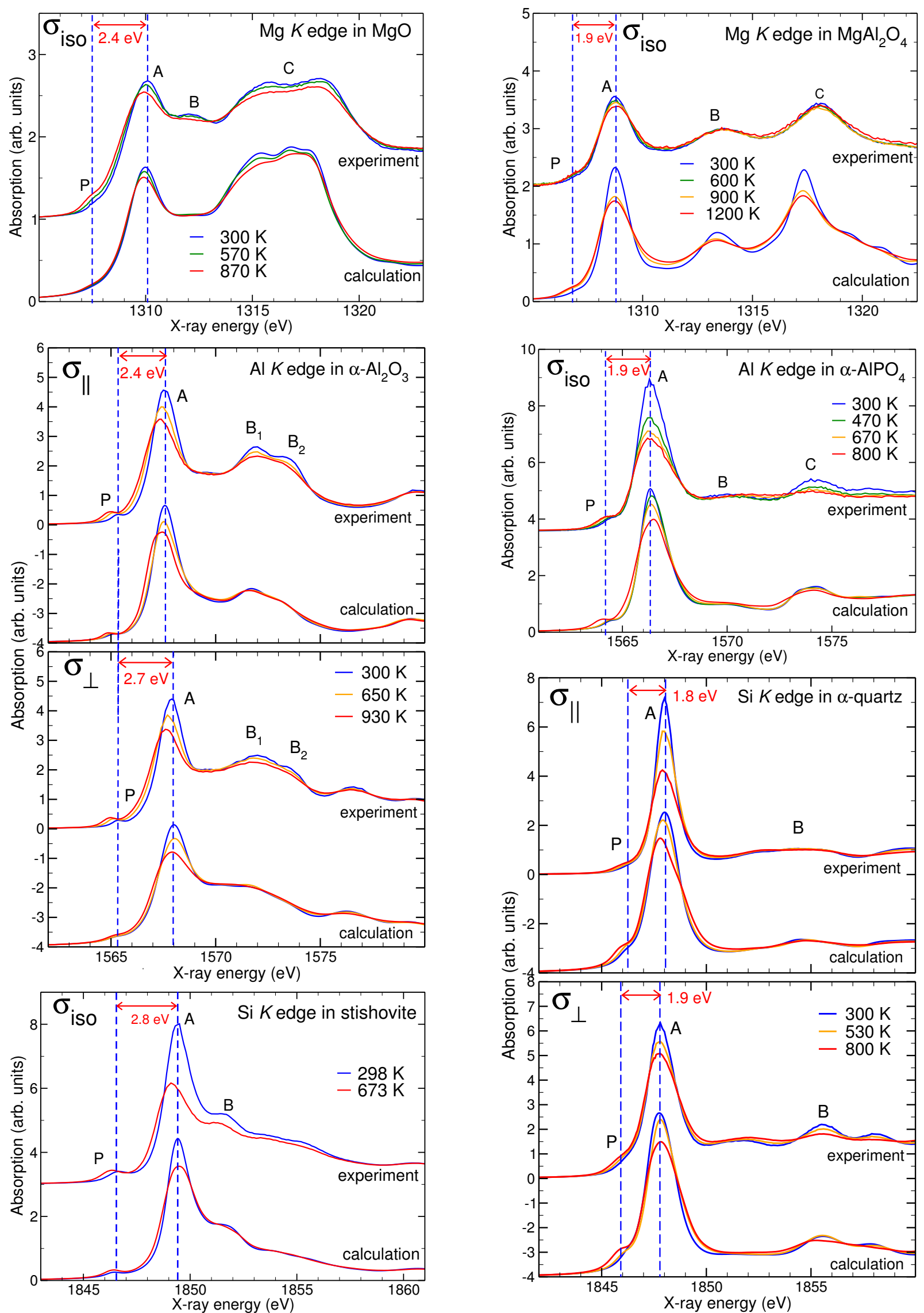

Fig. 2 Experimental and calculated temperature-dependent XANES spectra at the $K$ edges of $\mathrm{Mg}, \mathrm{Al}$ and $\mathrm{Si}$ in the mineral oxides, where the probed cation is either 6 (leftside) or 4 -fold coordinated (rightside) to oxygen. The isotropic spectra $\sigma_{\text {iso }}$ are shown for $\mathrm{MgO}_{1}$ stishovite, $\mathrm{MgAl}_{2} \mathrm{O}_{4}$ and $\alpha$ - $\mathrm{AlPO}_{4}$. When available, polarized spectra, $\sigma_{\perp}$ and $\sigma_{/ /}$, are displayed. The vertical dashed lines point to $\mathrm{P}$ and $\mathrm{A}$ peak energy positions. 


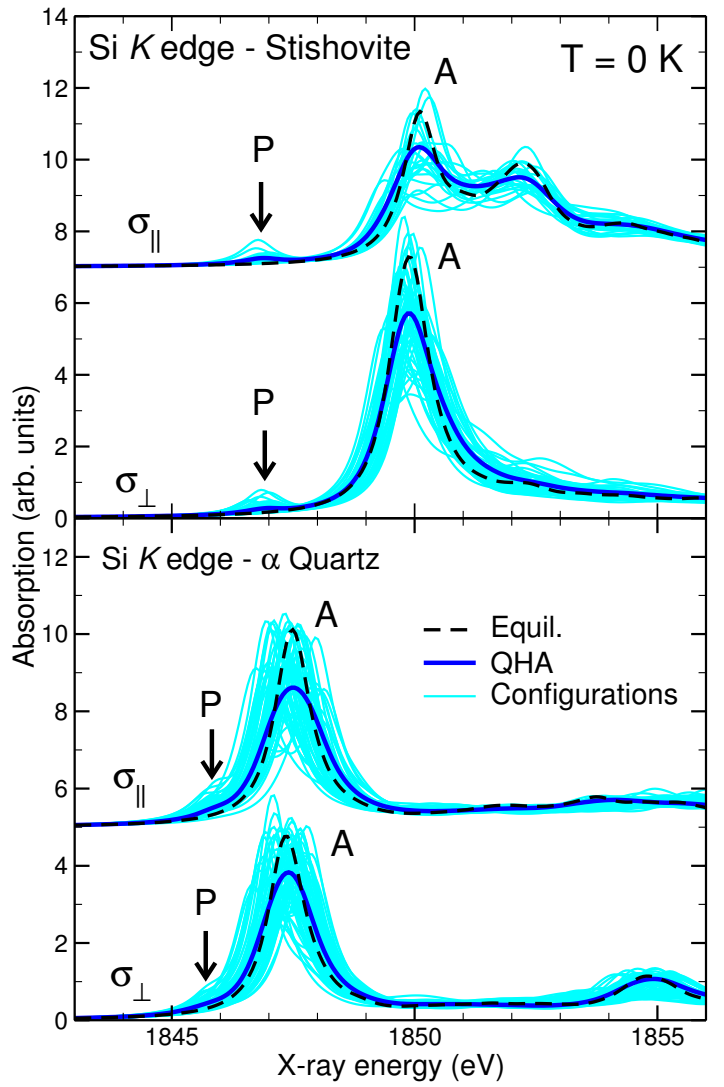

Fig. 3 Calculated Si $K$-edge XANES spectra in stishovite and $\alpha$-quartz $\left(\mathrm{SiO}_{2}\right.$ polymorphs) at $0 \mathrm{~K}$, for the $\sigma_{\perp}$ and $\sigma_{/ /}$orientations. The thick-solid spectra are averages of all configuration spectra (light) and dashed spectra are calculated in the equilibrium configurations. The energy positions are rescaled so that the theoretical and experimental main-edge peak energy match at room-temperature. The black arrows point out the pre-edge feature.

$\mathrm{Al}$ in $\mathrm{MgO}$ and $\alpha-\mathrm{Al}_{2} \mathrm{O}_{3}$ in Refs. 26 and 25, respectively. With temperature the $\mathrm{P}$ pre-edge peak grows while the A main-edge peak intensity decreases, P shifting towards lower energies. Highenergy XANES features are continually smoothed by temperature. As observed in NMR, a saturation mechanism is also expected at low temperature.

Fig. 3 displays the polarized Si $K$-edge XANES spectra calculated at $0 \mathrm{~K}$, in stishovite and $\alpha$-quartz, with all configuration spectra in background and compared to the equilibrium spectra. The latter are computed for atoms at their equilibrium positions in the 100-K XRD structure. The equilibrium calculation does not reproduce any pre-edge feature, unlike the QHA calculation. The presence of the $\mathrm{P}$ pre-edge peak at $0 \mathrm{~K}$ highlights the importance of accounting for quantum vibrations in XANES at low temperatures. Indeed, the zero-point motion, which is a pure quantum phenomenon, is sufficient to induce an $s-p$ hybridization resulting in the P peak occurrence. Fig. 3 also illustrates the angular dependance at $0 \mathrm{~K}$. In either x-ray polarization, the P-A separation is larger in stishovite than in $\alpha$-quartz. Figs. 2 and 3 show that the P pre-edge-peak intensity is similar in both orientations. Thus, there is no preferential structural orientation for the hybridization from which P originates. It is therefore complicated to propose geometrical arguments explaining the hybridization process, as already exposed in Ref. 26. The P pre-edge peak arises from a hybridization between the $3 s$ and $3 p$ electronic states of the absorbing atom induced by quantum vibrations. The increase of the P peak is the sign of a stronger hybridization with temperature. The energy shift is related to the band gap narrowing as temperature rises. ${ }^{26}$

\subsection{Sensitivity to the local environment}

The QHA model provides a satisfactory agreement between calculations and experiments that allows us to perform predictive computations when experimental data were not measured. Fig. 4 compares the calculated NMR chemical shifts for the cations in 4-fold (red triangles) and 6-fold coordinations, in the QHA model (full symbols) and by accounting for thermal expansion only (TEO, empty symbols). Opposite trends of QHA calculations are observed according to the coordination number of the probed cation. For 6-fold coordinated cations, the QHA corrected chemical shift increases with temperature, whereas it decreases for 4fold coordinated ones.

QHA and TEO calculations present distinct trends according to the cation coordination. For tetrahedral sites, QHA and TEO calculations give a decreasing chemical shift with temperature, with very similar slopes in the linear regime. On the other hand, in octahedral sites, there is an important deviation between QHA and TEO calculations, resulting in an increase of the QHA-calculated chemical shifts with temperature. These observations illustrate the competition between the nuclear dynamics and the thermal expansion. Nuclear dynamics is negligible compared to thermal expansion for 4-fold coordinated cations, whereas nuclear dynamics is the major component for 6-fold coordinated cations, and induces an increase of $\delta_{\text {iso }}$ experimentally observed in $\mathrm{MgO}$. This phenomenon probably originates from the local symmetry differences. Indeed, the bond lengths are longer in an octahedron than in a tetrahedron, giving more freedom to the 6-fold coordinated cations. For example, an analysis of the configurations at $300 \mathrm{~K}$ shows an average $0.07 \%$ off-center displacement in octahedra, whereas it reduces to $0.04 \%$ in tetrahedra. No significant variation in angles is observed.

It has already been observed that the chemical shift decreases when the cation-O bond length i.e., the tetrahedral volume increase. ${ }^{80-82}$ TEO calculations endorse this matter of fact. For $\mathrm{Mg}$ in $\mathrm{MgAl}_{2} \mathrm{O}_{4}$ and $\mathrm{Al}$ in $\alpha$ - $\mathrm{AlPO}_{4}$, the chemical shift decrease is about $3 \mathrm{ppm} \mathrm{K}^{-1}$, while it is less important for $\mathrm{Si}$ in $\alpha$-quartz about $0.5 \mathrm{ppm} \mathrm{K}^{-1}$. These differences are not directly related to the expansion of tetrahedra in each materials, since, from 300 to $800 \mathrm{~K}$, their volumes increase of about $1.55 \%$ for $\mathrm{MgO}_{4}, 2.91 \%$ for $\mathrm{AlO}_{4}$ and $2.53 \%$ for $\mathrm{SiO}_{4}$ units.

The calculated temperature-dependent NMR chemical shifts of ${ }^{17} \mathrm{O}$ in oxides are presented in Fig. 5, including both QHA and TEO calculations. Overall, the chemical shift varies significantly with temperature and the quantum nuclear dynamics influence is more important than in the TEO case. Interestingly, the most important variation occurs in ternary oxides $\left(\mathrm{MgAl}_{2} \mathrm{O}_{4}\right.$ and $\alpha-\mathrm{AlPO}_{4}$ ), possibly due to the fact that oxygen atoms are sur- 

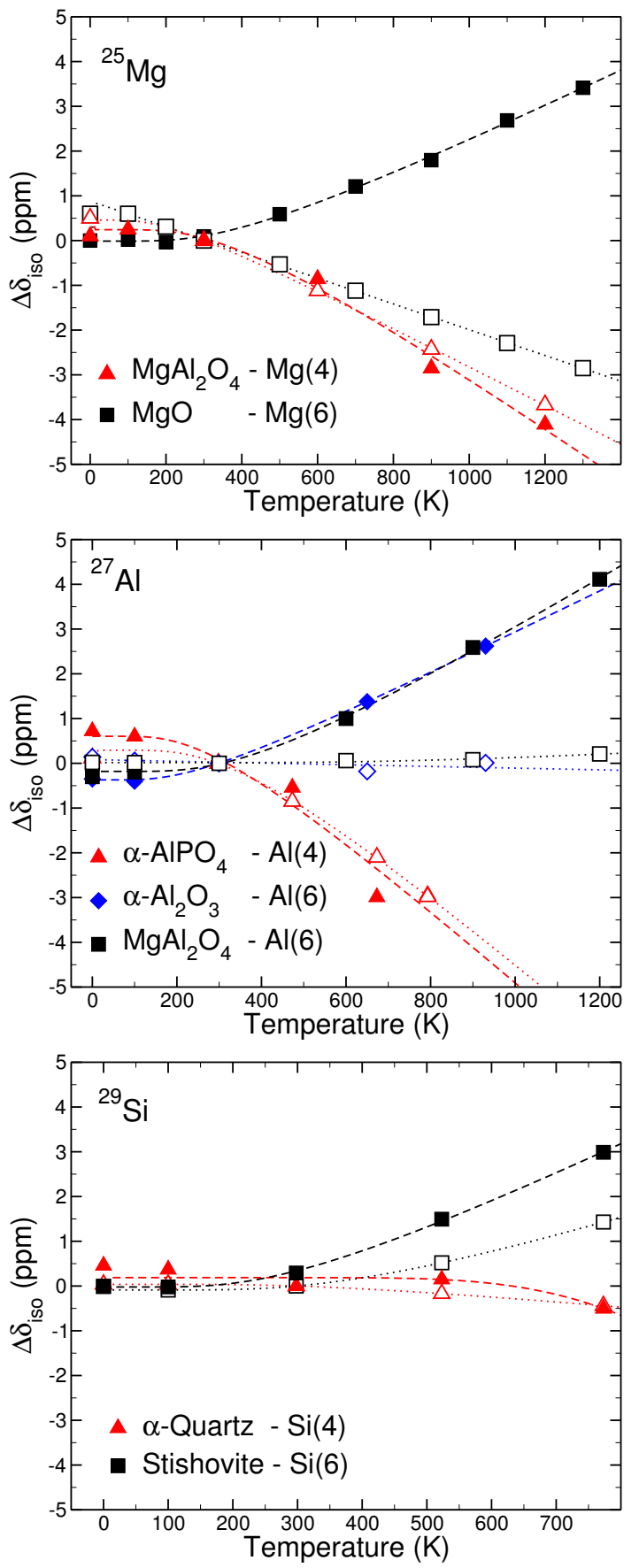

Fig. 4 Calculated temperature-dependent NMR chemical shifts of the studied cations in oxides, where the absorbing atom is either 4- or 6-fold coordinated. Black squares and blue diamonds are used for cations in octahedral environment. Red triangles represent the cation in tetrahedral environments, the full and empty symbols correspond to the QHA and TEO (thermal expansion only) calculations, respectively. The dashed lines are the best fits using eqn (11). Saturation temperatures, $T_{s}$, are given in ESI. ${ }^{\dagger}$

rounded by different atom-type neighbors.

In XANES spectroscopy, the temperature-dependence is also sensitive to the probed cation coordination number. For octahedral environments, earlier studies demonstrated that the P peak observable at the $K$ pre-edge of light-cation XANES spectra orig-

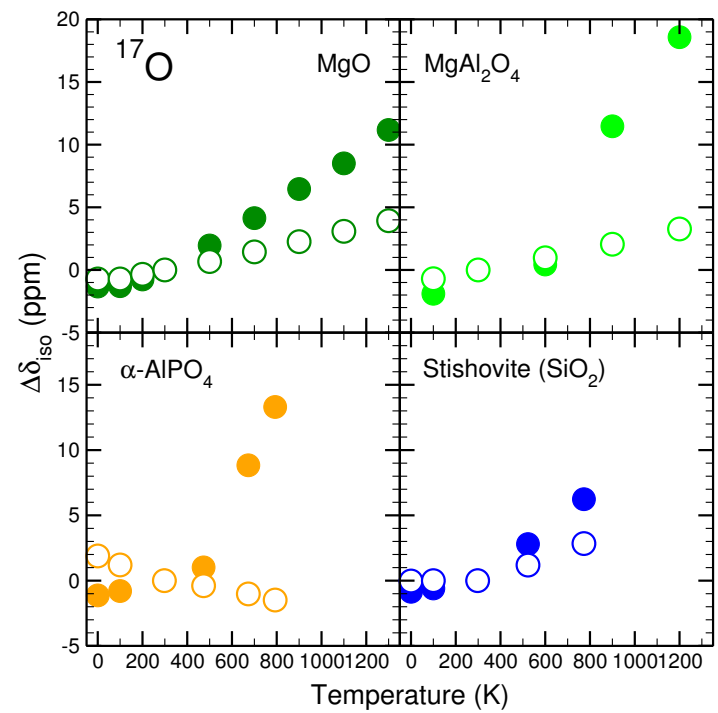

Fig. 5 Calculated temperature-dependent NMR chemical shifts of ${ }^{17} \mathrm{O}$ in oxides. Full and empty symbols dedicates to the QHA and TEO calculations, respectively.

inates from $1 s$ to $3 s$-like transitions allowed by $s-p$ hybridizations induced by quantum vibrations. ${ }^{17,25,26}$ The appearance of the P pre-edge peak at the $K$ edges of atoms in tetrahedral environments shows that a similar mechanism occurs, but some distinctions are observed between the 4- and 6-fold cases. Fig. 2 shows that at the $K$ edge of 4 -fold coordinated atoms, the A peak energy position is systematically located at lower energies than for 6-fold coordinated cations. This well-known phenomenon is commonly used to identify chemical environments in unknown species. ${ }^{83-85}$ The $\mathrm{P}$ pre-edge peak is less discernable in tetrahedral symmetry given that the P-A energy distance is only about $1.8 \mathrm{eV}$ while in octahedral symmetry it is about $2.5 \mathrm{eV}$. The experimental $\mathrm{P}$ energy variations as a function of temperature are displayed in Fig. 6. When the probed atom lies in an octahedral site, the $\mathrm{P}$ peak shifts towards lower energies are larger than in tetrahedral sites. These shifts can be related to variations of elec-

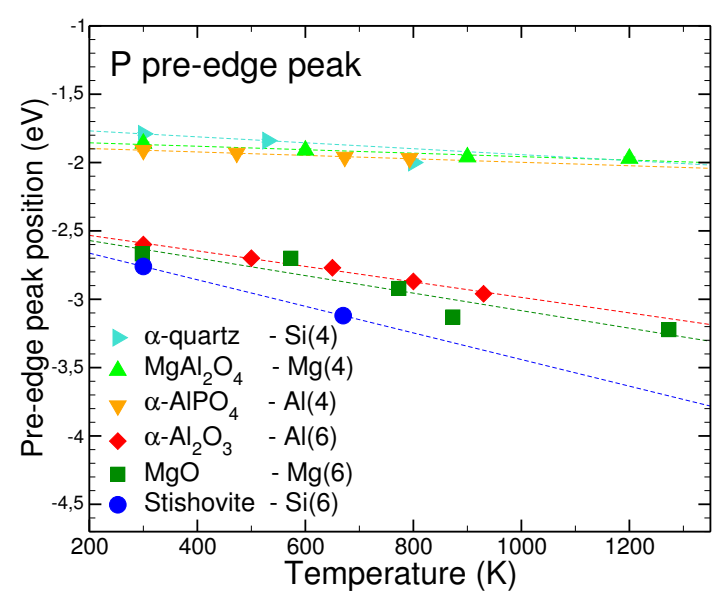

Fig. 6 Experimental energy positions of the P pre-edge peak as a function of temperature. The energy positions are given with respect to the A peak position at $300 \mathrm{~K}$. 
tronic band gaps with temperature. Using optical measurements, the electronic band gap was found to decrease by $0.91 \mathrm{eV}$ in $\mathrm{MgO}$, $1.0 \mathrm{eV}$ in $\mathrm{Al}_{2} \mathrm{O}_{3}$ and only $0.78 \mathrm{eV}$ in $\mathrm{MgAl}_{2} \mathrm{O}_{4}$, where cations are both 4 and 6-fold coordinated. ${ }^{86-88}$

\subsection{Quadrupolar coupling constant in temperature}

In quadrupolar nuclei, quadrupolar interaction between $Q$ nuclear quadrupole moment and the electric-field gradient at the nucleus generated by the other charges in the system must be taken into account. Hence, there is much structural information to be obtained from the $C_{Q}$ quadrupolar coupling parameters. The computed $C_{Q}$ for quadrupolar nuclei $\left({ }^{25} \mathrm{Mg},{ }^{27} \mathrm{Al}\right.$ and ${ }^{17} \mathrm{O}$ ) are reported in Fig. 7. In the equilibrium configuration of $\mathrm{MgAl}_{2} \mathrm{O}_{4}$ and $\mathrm{MgO}$, the ${ }^{25} \mathrm{Mg} C_{Q}$ vanishes due to symmetry arguments. ${ }^{89}$ In $\mathrm{MgO}$, the dynamic disorder induced by quantum thermal vibrations does not produce any quadrupolar coupling, whereas in $\mathrm{MgAl}_{2} \mathrm{O}_{4}$ the $C_{Q}$ varies with temperature, as observed for all other nuclei. This observation provides an explanation of the overestimation of the ${ }^{17} \mathrm{O}-C_{Q}$ in equilibrium calculations. ${ }^{90}$ The temperature-dependence of quadrupole resonance frequencies was investigated in the 1950s by nuclear quadrupolar resonance measurements of ${ }^{35} \mathrm{Cl}$ in $\mathrm{KClO}_{3}$ and ${ }^{63} \mathrm{Cu}$ in $\mathrm{Cu}_{2} \mathrm{O} .{ }^{91}$ The observed trends were explained by a dependence to the volume variation and strain deformations induced by thermal vibrations. By describing vibrations at the quantum harmonic level with analytical expressions, Wang ${ }^{92}$ showed that when the vibration amplitude increases, the quadrupolar coupling decreases. However, the present calculations prove that theses trends are not systematic. Moreover, the computation of the quadrupolar coupling of deuterium in hydrogen-bounded compounds using Car-Parrinello molecular dynamics ${ }^{93}$ demonstrated that, in some cases, the unexpected augmentation of the quadrupolar coupling arises from anharmonic vibrations. ${ }^{68}$

\section{Conclusions}

The combination of experimental and theoretical investigations permits to investigate the effect of temperature on NMR and XANES spectra of light-element oxides. The presence of the preedge peak in the $K$-edge XANES spectra and the variations of NMR resonance values are relevant signatures of the influence of quantum thermal vibrations. These observations result from both contributions of thermal expansion and quantum nuclear vibrations. In both cases, the experiment-calculation agreement is very good.

In NMR, linear extrapolations to $0 \mathrm{~K}$ are inappropriate to predict temperature-dependent NMR parameters. Thus, thermal effect must be considered using a relevant theoretical methodology even at low temperatures. This is a crucial issue as with the extension of variable-temperature XANES and NMR measurements, such as in the field of catalyst, it is mandatory to discriminate features coming from thermal vibration effects than from the reaction itself.

In XANES, an unexpected pre-edge peak is observed at the $K$ edge of light cations. This feature originates from electronic transitions allowed by $s-p$ hybridizations induced by quantum vibrations. The presence of such feature starting from $0 \mathrm{~K}$ high- lights the importance of quantum vibration effects in XANES spectroscopy.

The influence of the local symmetry of the cation on the occurrence of thermal effects is brought to light. In both techniques, the impact of vibrations is more important when the probed atom lies in an octahedral environment. In XANES, the additional pre-edge features and energy shifts are stronger and in NMR, the chemical shifts deviate greatly from the thermal expansion scheme in 6-fold coordinated compounds. On the other hand, experimental data are dominated by lattice thermal expansion in tetrahedral environments.

Further experimental measurements are expected to assess the NMR and nuclear quadrupolar resonance predictive calculations provided in this paper. As it is, this methodology is perfectly suitable to investigate more complicated systems such as zeolites or hybrid materials, of interest for physicists, chemists and Earth scientists.

\section{Acknowledgements}

This work was supported by French state funds managed by the ANR within the Investissements d'Avenir programme under reference ANR-11-IDEX-0004-02, and more specifically within the framework of the Cluster of Excellence MATISSE led by Sorbonne Universités. Computer facilities were provided by GENCI-IDRIS (Project No. 2015100172). We acknowledge SOLEIL for provision of synchrotron radiation facilities and we would like to thank the staff of the LUCIA beamline (Proposal 20141057) and "IR RMN THC FR 3050". The authors thank L. Verger for synthesizing the spinel powder sample, A. Shatskiy for the high-pressure synthesis of the stishovite sample and E. Balan for fruitful discussions.

\section{References}

1 G. S. Henderson, F. M. F. de Groot and B. J. A. Moulton, Rev. Mineral. Geochem., 2014, 78, 75-138.

2 J. F. Stebbins and X. Xue, Rev. Mineral. Geochem., 2014, 78, 605-653.

3 A. Jokic, J. N. Cutler, E. Ponomarenko, G. van der Kamp and D. W. Anderson, Geochim. Cosmochim. Acta, 2003, 67, 25852597.

4 D. R. Neuville, L. Cormier and D. Massiot, Geochim. Cosmochim. Acta, 2004, 68, 5071-5079.

5 A. Vjunov, J. L. Fulton, T. Huthwelker, S. Pin, D. Mei, G. K. Schenter, N. Govind, D. M. Camaioni, J. Z. Hu and J. A. Lercher, J. Am. Chem. Soc., 2014, 136, 8296-8306.

6 A. Lesage, Phys. Chem. Chem. Phys., 2009, 11, 6876.

7 E. Salager, R. S. Stein, S. Steuernagel, A. Lesage, B. Elena and L. Emsley, Chem. Phys. Lett., 2009, 469, 336-341.

8 S. E. Ashbrook, Phys. Chem. Chem. Phys., 2009, 11, 6892.

9 C. J. Milne, T. J. Penfold and M. Chergui, Coord. Chem. Rev., 2014, 277-278, 44-68.

10 A. Koide, T. Fujikawa and N. Ichikuni, J. Electron Spectrosc. Relat. Phenom., 2014, 195, 375-381.

11 A. Bordage, V. Trannoy, O. Proux, H. Vitoux, R. Moulin and 

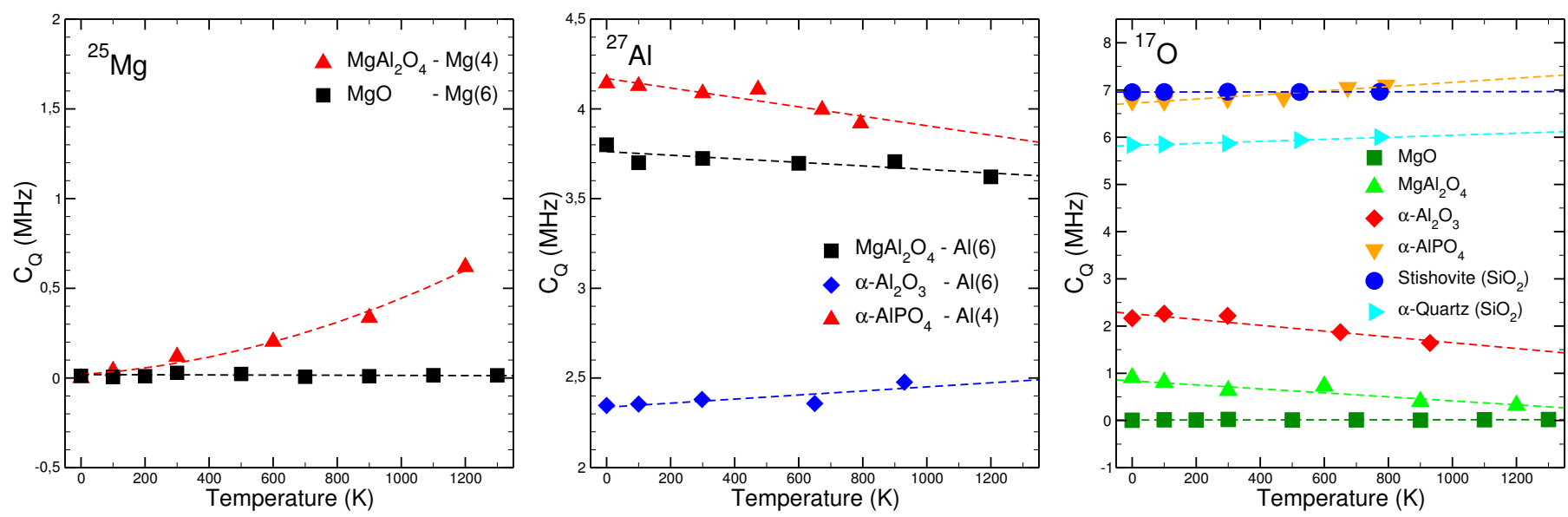

Fig. 7 Temperature dependence of $C_{Q}$ for ${ }^{25} \mathrm{Mg},{ }^{27} \mathrm{Al}$ and ${ }^{17} \mathrm{O}$. Red triangles represent tetrahedral environments. Dashed lines are displayed as a guide for eyes.

A. Bleuzen, Phys. Chem. Chem. Phys., 2015, 17, 1726017265.

12 J. J. Rehr and R. C. Albers, Rev. Mod. Phys., 2000, 72, 621654.

13 S. E. Ashbrook, L. Le Pollès, C. J. Pickard, A. J. Berry, S. Wimperis and I. Farnan, Phys. Chem. Chem. Phys., 2007, 9, 1587.

14 T. Charpentier, Solid State Nucl. Magn. Reson., 2011, 40, 120.

15 C. Bonhomme, C. Gervais, F. Babonneau, C. Coelho, F. Pourpoint, T. Azaïs, S. E. Ashbrook, J. M. Griffin, J. R. Yates, F. Mauri and C. J. Pickard, Chem. Rev., 2012, 112, 57335779.

16 M. Ducher, M. Blanchard, D. Vantelon, R. Nemausat and D. Cabaret, Phys. Chem. Miner., 2016, 43, 217-227.

17 D. Cabaret and C. Brouder, J. Phys.: Conf. Ser., 2009, 190, 012003.

18 S. Sene, M. Reinholdt, G. Renaudin, D. Berthomieu, C. M. Zicovich-Wilson, C. Gervais, P. Gaveau, C. Bonhomme, Y. Filinchuk, M. E. Smith, J.-M. Nedelec, S. Bégu, P. H. Mutin and D. Laurencin, Chem. - Eur. J., 2013, 19, 880-891.

19 M. Ostafin and B. Nogaj, Measurement, 2007, 40, 43-54.

20 A. L. Webber, B. Elena, J. M. Griffin, J. R. Yates, T. N. Pham, F. Mauri, C. J. Pickard, A. M. Gil, R. Stein, A. Lesage, L. Emsley and S. P. Brown, Phys. Chem. Chem. Phys., 2010, 12, 6970.

21 I. D. Gortari, G. Portella, X. Salvatella, V. S. Bajaj, P. C. A. van der Wel, J. R. Yates, M. D. Segall, C. J. Pickard, M. C. Payne and M. Vendruscolo, J. Am. Chem. Soc., 2010, 132, 5993-6000.

22 L. A. O’Dell, R. W. Schurko, K. J. Harris, J. Autschbach and C. I. Ratcliffe, J. Am. Chem. Soc., 2011, 133, 527-546.

23 C. E. Patrick and F. Giustino, Nat. Commun., 2013, 4, 2006.

24 S. Nozawa, T. Iwazumi and H. Osawa, Phys. Rev. B: Condens. Matter Mater. Phys., 2005, 72, 121101.

25 D. Manuel, D. Cabaret, C. Brouder, P. Sainctavit, A. Bordage and N. Trcera, Phys. Rev. B: Condens. Matter Mater. Phys., 2012, 85, 224108.

26 R. Nemausat, D. Cabaret, C. Gervais, C. Brouder, N. Trcera,
A. Bordage, I. Errea and F. Mauri, Phys. Rev. B: Condens. Matter Mater. Phys., 2015, 92, 144310.

27 P. S. Fiske, J. F. Stebbins and I. Farnan, Phys. Chem. Miner., 1994, 20, 587-593.

28 C. Bonhomme, C. Gervais, C. Coelho, F. Pourpoint, T. Azaïs, L. Bonhomme-Coury, F. Babonneau, G. Jacob, M. Ferrari, D. Canet, J. R. Yates, C. J. Pickard, S. A. Joyce, F. Mauri and D. Massiot, Magn. Reson. Chem., 2010, 48, S86-S102.

29 D. Bräunling, O. Pecher, D. M. Trots, A. Senyshyn, D. A. Zherebtsov, F. Haarmann and R. Niewa, Z. Anorg. Allg. Chem., 2010, 636, 936-946.

30 A. L. Ankudinov and J. J. Rehr, Phys. Scr., T, 2005, T115, 24.

31 C. Gervais, L. Bonhomme-Coury, F. Mauri, F. Babonneau and C. Bonhomme, Phys. Chem. Chem. Phys., 2009, 11, 69536961.

32 N. Folliet, C. Roiland, S. Bégu, A. Aubert, T. Mineva, A. Goursot, K. Selvaraj, L. Duma, F. Tielens, F. Mauri, G. Laurent, C. Bonhomme, C. Gervais, F. Babonneau and T. Azaïs, J. Am. Chem. Soc., 2011, 133, 16815-16827.

33 J. S. Uejio, C. P. Schwartz, R. J. Saykally and D. Prendergast, Chem. Phys. Lett., 2008, 467, 195-199.

34 J.-N. Dumez and C. J. Pickard, J. Chem. Phys., 2009, 130, 104701.

35 M. Robinson and P. D. Haynes, J. Chem. Phys., 2010, 133, 084109.

36 A. H. England, A. M. Duffin, C. P. Schwartz, J. S. Uejio, D. Prendergast and R. J. Saykally, Chem. Phys. Lett., 2011, 514, 187-195.

37 T. A. Pascal, U. Boesenberg, R. Kostecki, T. J. Richardson, T.C. Weng, D. Sokaras, D. Nordlund, E. McDermott, A. Moewes, J. Cabana and D. Prendergast, J. Chem. Phys., 2014, 140, 034107.

38 C. P. Schwartz, J. S. Uejio, R. J. Saykally and D. Prendergast, J. Chem. Phys., 2009, 130, 184109.

39 M. Dračínský, P. BouÅź and P. Hodgkinson, J. Chem. Theory Comput., 2016, 12, 968-973.

40 G. J. O. Beran, J. D. Hartman and Y. N. Heit, Acc. Chem. Res., 
2016, 49, 2501-2508.

41 G. J. O. Beran, Chem. Rev., 2016, 116, 5567-5613.

42 R. O. Jones, Rev. Mod. Phys., 2015, 87, 897-923.

43 S. Rossano, F. Mauri, C. J. Pickard and I. Farnan, J. Phys. Chem. B, 2005, 109, 7245-7250.

44 S. Baroni, P. Giannozzi and E. Isaev, Rev. Mineral. Geochem., 2010, 71, 39-57.

45 R. Nemausat, $P h D$ thesis, Université Pierre et Marie Curie, 2016.

46 C. Brouder, J. Phys.: Condens. Matter, 1990, 2, 701-738.

47 R. Hazen, Am. Mineral., 1976, 61, 266-271.

48 R. C. Peterson, G. A. Lager and R. L. Hitterman, Am. Mineral., 1991, 76, 1455-1458.

49 G. Fiquet, P. Richet and G. Montagnac, Phys. Chem. Miner., 1999, 27, 103-111.

50 M. Lucht, M. Lerche, H.-C. Wille, Y. V. Shvyd'ko, H. D. Rüter, E. Gerdau and P. Becker, J. Appl. Crystallogr., 2003, 36, 10751081.

51 Y. Muraoka and K. Kihara, Phys. Chem. Miner., 1997, 24, 243253.

52 H. Ito, K. Kawada and S.-I. Akimoto, Phys. Earth Planet. Inter., 1974, 8, 277-281.

53 G. A. Lager, J. D. Jorgensen and F. J. Rotella, J. Appl. Phys. (Melville, NY, U. S.), 1982, 53, 6751.

54 K. Kihara, Eur. J. Mineral., 1990, 2, 63-77.

55 P. Giannozzi, S. Baroni, N. Bonini, M. Calandra, R. Car, C. Cavazzoni, D. Ceresoli, G. L. Chiarotti, M. Cococcioni, I. Dabo, A. Dal Corso, S. de Gironcoli, S. Fabris, G. Fratesi, R. Gebauer, U. Gerstmann, C. Gougoussis, A. Kokalj, M. Lazzeri, L. Martin-Samos, N. Marzari, F. Mauri, R. Mazzarello, S. Paolini, A. Pasquarello, L. Paulatto, C. Sbraccia, S. Scandolo, G. Sclauzero, A. P. Seitsonen, A. Smogunov, P. Umari and R. M. Wentzcovitch, J. Phys.: Condens. Matter, 2009, 21, 395502.

56 S. Baroni, S. de Gironcoli, A. Dal Corso and P. Giannozzi, Rev. Mod. Phys., 2001, 73, 515-548.

57 X. Gonze, Phys. Rev. A, 1995, 52, 1096-1114.

58 M. Taillefumier, D. Cabaret, A. M. Flank and F. Mauri, Phys. Rev. B: Condens. Matter Mater. Phys., 2002, 66, 195107.

59 C. Gougoussis, M. Calandra, A. P. Seitsonen and F. Mauri, Phys. Rev. B: Condens. Matter Mater. Phys., 2009, 80, 075102.

60 C. J. Pickard and F. Mauri, Phys. Rev. B: Condens. Matter Mater. Phys., 2001, 63, 245101.

61 J. R. Yates, C. J. Pickard and F. Mauri, Phys. Rev. B: Condens. Matter Mater. Phys., 2007, 76, 024401.

62 I. Errea, M. Calandra and F. Mauri, Phys. Rev. Lett., 2013, 111, 177002

63 I. Errea, M. Calandra and F. Mauri, Phys. Rev. B: Condens. Matter Mater. Phys., 2014, 89, 064302.

64 J. P. Perdew, K. Burke and M. Ernzerhof, Phys. Rev. Lett., 1996, 77, 3865.

65 N. Troullier and J. L. Martins, Phys. Rev. B: Condens. Matter Mater. Phys., 1991, 43, 1993.
66 K. F. Garrity, J. W. Bennett, K. M. Rabe and D. Vanderbilt, Comput. Mater. Sci., 2014, 81, 446-452.

67 P. Pyykkö, Mol. Phys., 2008, 106, 1965-1974.

68 J. Schmidt and D. Sebastiani, J. Chem. Phys., 2005, 123, 074501.

69 T. Mizoguchi, I. Tanaka, S.-P. Gao and C. J. Pickard, J. Phys.: Condens. Matter, 2009, 21, 104204.

70 E. Gaudry, A. Kiratisin, P. Sainctavit, C. Brouder, F. Mauri, A. Ramos, A. Rogalev and J. Goulon, Phys. Rev. B: Condens. Matter Mater. Phys., 2003, 67, 094108.

71 Two stishovite polycristalline samples were synthesized under $14 \mathrm{GPa}$ and $1900 \mathrm{~K}$ conditions for 2 hours, using a multianvil press. The synthesis was directed by Dr Anton Shatskiy at Tohoku University, Sendai (Japan).

72 R. A. Shakhovoy, V. Sarou-Kanian, A. Rakhmatullin, E. Véron and C. Bessada, J. Appl. Phys. (Melville, NY, U. S.), 2015, 118, 243906.

73 D. Vantelon, N. Trcera, D. Roy, T. Moreno, D. Mailly, S. Guilet, E. Metchalkov, F. Delmotte, B. Lassalle, P. Lagarde and A.-M. Flank, J. Synchrotron Radiat., 2016, 23, 635-640.

74 R. Nemausat, C. Brouder, C. Gervais and D. Cabaret, J. Phys.: Conf. Ser., 2016, 712, 012006.

75 L. He, F. Liu, G. Hautier, M. J. T. Oliveira, M. A. L. Marques, F. D. Vila, J. J. Rehr, G.-M. Rignanese and A. Zhou, Phys. Rev. B: Condens. Matter Mater. Phys., 2014, 89, 064305.

76 Z. Wu and R. E. Cohen, Phys. Rev. B: Condens. Matter Mater. Phys., 2006, 73, 235116.

77 J. P. Perdew, A. Ruzsinszky, G. I. Csonka, O. A. Vydrov, G. E. Scuseria, L. A. Constantin, X. Zhou and K. Burke, Phys. Rev. Lett., 2008, 100, 136406.

78 E. K. H. Salje, B. Wruck and H. Thomas, Z. Phys. B: Condens Matter, 1991, 82, 399-404.

79 E. Balan, S. Delattre, M. Guillaumet and E. K. H. Salje, Am. Mineral., 2010, 95, 1257-1266.

80 T. M. Clark, P. J. Grandinetti, P. Florian and J. F. Stebbins, Phys. Rev. B: Condens. Matter Mater. Phys., 2004, 70, 064202

81 L. Cahill, J. V. Hanna, A. Wong, J. C. C. Freitas, J. R. Yates, R. K. Harris and M. E. Smith, Chem. - Eur. J., 2009, 15, 97859798.

82 E. Gambuzzi, A. Pedone, M. C. Menziani, F. Angeli, D. Caurant and T. Charpentier, Geochim. Cosmochim. Acta, 2014, 125 170-185.

83 A. Mottana, J.-L. Robert, A. Marcelli, G. Giuli, G. Della Ventura, E. Paris and Wu Ziyu, Am. Mineral., 1997, 82, 497-502.

84 P. Ildefonse, D. Cabaret, P. Sainctavit, G. Calas, A. M. Flank and P. Lagarde, Phys. Chem. Miner., 1998, 25, 112-121.

85 J. A. van Bokhoven, T. Nabi, H. Sambe, D. E. Ramaker and D. C. Koningsberger, J. Phys.: Condens. Matter, 2001, 13, 10247-10260.

86 M. L. Bortz, R. H. French, D. J. Jones, R. V. Kasowski and F. S. Ohuchi, Phys. Scr., T, 1990, 41, 537-541.

87 R. H. French, R. V. Kasowski, F. S. Ohuchi, D. J. Jones, H. Song and R. L. Coble, J. Am. Ceram. Soc., 1990, 73, 3195-99.

88 W. L. O’Brien, J. Jia, Q.-Y. Dong, T. A. Callcott, D. R. Mueller 
and D. L. Ederer, Phys. Rev. B: Condens. Matter Mater. Phys., 1992, 45, 3882.

89 P. J. Pallister, I. L. Moudrakovski and J. A. Ripmeester, Phys. Chem. Chem. Phys., 2009, 11, 11487-11500.

90 C. Gervais, R. Dupree, K. J. Pike, C. Bonhomme, M. Profeta, C. J. Pickard and F. Mauri, J. Phys. Chem. A, 2005, 109, 6960-
6969.

91 T. Kushida, G. B. Benedek and N. Bloembergen, Phys. Rev., 1956, 104, 1364-1377.

92 T.-C. Wang, Phys. Rev., 1955, 99, 566-577.

93 J. Hutter, Comput. Mol. Sci., 2012, 2, 604-612. 